\title{
ANALYSIS OF APPLICABILITY OF TOOLS OF INVENTORY CONTROL AND KNOWLEDGE OF HOSPITAL PHARMACISTS ON METHODS OF INVENTORY IN THE CENTRAL PHARMACY OF A TERTIARY CARE HOSPITAL
}

\author{
SAI ASHWINI R. ${ }^{1}$, SAI CHOWDRY B. ${ }^{2}$, SAI SANNIHITHA B. ${ }^{3}$, SENTHIL RAJ V. ${ }^{4}$, VANITHA RANI N. ${ }^{*}$, THENNARASU \\ P. ${ }^{5}$, MALARVIZHI AIYAVOO ${ }^{6}$
}

1,2,3,4Pharm D, Faculty of Pharmacy, Sri RamachandraInstitute of Higher Education and Research (Deemed to be University), Chennai,

${ }^{*}$ Department of Pharmacy Practice, Faculty of Pharmacy, Sri RamachandraInstitute of Higher Education and Research (Deemed to be University), Chennai, ${ }^{6}$ Chief Pharmacy Administrator, Sri Ramachandra Medical Center and Hospital, Chennai

Email: reachvanitha99@gmail.com

Received: 04 Feb 2019 Revised and Accepted: 21 Jun 2019

\begin{abstract}
Objective: A study was conducted to analyse the applicability of the tools of inventory control of drugs, and the awareness of hospital pharmacists on the inventory tools in the central pharmacy of a tertiary care hospital.

Methods: Always Better Control (ABC) and High Moderate Low (HML) analysis were carried out for 4057 drugs purchased in the central pharmacy during the financial year of 2017-2018. The drugs were classified as A, B and C category based on their annual usage value and as Category High (H), Moderate (M) and low (L) drugs based on their unit price respectively. The knowledge of the 80 hospital pharmacists on the tools of inventory control was assessed using a structured questionnaire.

Results: Based on $\mathrm{ABC}$ analysis, of 4057 drugs, 330 drugs were classified under "A" category accounting to $8.13 \%$ of the total number of drugs purchased which was $70.91 \%$ of total annual consumption in money value. The category "B" consisted of 667 drugs accounting to $16.44 \%$ of the total number of drugs which was $20.01 \%$ of total annual money consumption. Category "C" had 3060 drugs which accounted to $75.42 \%$ of the total number of drugs purchased which reflected on 9.01\% of annual money consumption. Based on HML analysis, 440 drugs (10\%) had a unit price above Rs. 500 and were classified under ' $\mathrm{H}$ ' category. The drugs with unit price between Rs.500 and Rs.100 were grouped as ' $\mathrm{M}$ ' items. There were 695 drugs (17.13\%) in 'M' category. The ' $L$ ' category consisted of 2922 drugs (72.02\%) with their unit price below Rs.100. Of 80 pharmacists, $84 \%$ had good overall knowledge on the inventory control tools and 59 participants were willing to acquire additional training on inventory control techniques.
\end{abstract}

Conclusion: The study explained the need for the application of ABC and HML analysis, and the involvement of hospital pharmacists in the regular implementation of inventory analysis towards effective management of Pharmacy stores in a hospital setting.

Keywords: Inventory control, ABC analysis, HML analysis

(C) 2019 The Authors. Published by Innovare Academic Sciences Pvt Ltd. This is an open-access article under the CC BY license (http://creativecommons.org/licenses/by/4.0/) DOI: http://dx.doi.org/10.22159/ijpps.2019v11i8.32390

\section{INTRODUCTION}

Pharmacy stores are very essential supportive services of any hospital and as the hospital grows in size the need of a pharmacy store also increases [1]. Hospital supply systems should ensure adequate stock of all the required items to maintain uninterrupted supply. There is a need for planning, designing and organizing the pharmacy in a manner that results in efficient clinical and administrative services [2-3].

Hospital pharmacy inventory control is very important in a developing country like India [4]. The cost containment and improved efficiency should be stressed for hospital drug inventory management [5]. Every drug item may be considered critical and there is a professed need to supply very high levels of service [6]. The stocking of hospital pharmacy items can be expensive and the majority of hospital capital is held up in these items and it can be reduced by $30-40 \%$ of a hospital's budget by bringing efficiencies to important cost drivers. Since inventory constitutes a major segment of total investment, it is crucial that good inventory management is practiced to ensure growth and profitability [7].

In pharmacy operations, inventory is referred to as the stock of pharmaceutical products retained to meet future demand. Inventory represents the largest current asset, as well as liquid asset in pharmacy practice and its value, continues to rise because of the growth in variety and cost of pharmaceutical products. Inventory Control focuses on the process of movement and accountability of inventory. This consists of strict policies and processes in regards to physical and systemic movement of materials, physical inventory and cycle counting, measurement of accuracy and tolerances, and good accounting practices [8].

Inventory Management in its broadest perspective is to keep the most economical amount of one kind of asset in order to facilitate an increase in the total value of all assets of the organization-human and material resources [9]. The objective of inventory management is to determine how much of an inventory to re-order, when to re-order the inventory, how frequently orders should be placed and what the appropriate safety stock is, for minimizing stock-outs, and at low cost [10]

$\mathrm{ABC}$ analysis is based on Pareto principle (80-20 rule) which states that $80 \%$ of the overall consumption value (expense) is based only on $20 \%$ of the total items, i.e. small portion of the items may typically represent the bulk of money value, while a relatively large number of items may form a small part of the money value. $\mathrm{ABC}$ analysis is a method for dividing the theon-hand inventory into three classifications $\mathrm{A}, \mathrm{B}, \mathrm{C}$ based on annual consumption unit." $A$ " items: requiring tight inventory control under more experienced management. Re-orders should be more frequent. " $\mathrm{B}$ " items: requiring medium attention for control. " $\mathrm{C}$ " items: requiring minimum attention and maybe kept under simple observation. Reordering is less frequent [11-13].

The HML classification is generally done based on the weight of the items like 'heavyweight', 'medium weight' and 'lightweight'. This classification is useful in the storage and display of items in the stores. $\mathrm{H}$ stands for high priced items; $\mathrm{M}$ for Medium priced items and $\mathrm{L}$ denotes low priced items. HML classification may also be done 
based on the unit value of individual items. In this concept, items are grouped as high cost, medium cost and low cost. An analysis similar to the one in $\mathrm{ABC}$ is done. HML analysis helps to assess storage and security requirements, i.e. storage of the high priced items in the cupboards and execute control over consumption as per the price, thus outline the buying policies of drugs [14-16]. The present study was conducted to analyse the effectiveness and applicability of $A B C$ and HML tools for the inventory of drugs and to identify the item categories requiring greater supervisory monitoring in the central pharmacy of a tertiary care hospital.

\section{MATERIALS AND METHODS}

\section{Study design}

A hospital-based study was conducted after obtaining approval from the Institutional Ethics committee by using quantitative data collection methods to analyse the applicability and the effectiveness of $\mathrm{ABC}$ and HML inventory control techniques towards the management of the inventory of the hospital pharmacy by applying
$\mathrm{ABC}$ and HML methods of inventory analysis on the annual consumption of items and expenditure incurred in the pharmacy store during the financial year April 2017-March 2018. The study also assessed the knowledge of 80 hospital pharmacists on methods of inventory control.

\section{Data collection}

Data on the details of the drugs procured in the central pharmacy during the financial year (April 2017-March 2018) were extracted from the hospital electronic database.

\section{Always better control (ABC) analysis}

\section{Method I}

The data of annual demand and expenditure incurred on each drug in the hospital's central pharmacy for the financial year 2017-2018 was collected. The data was transcribed in an MS Excel spreadsheet and the $\mathrm{ABC}$ analysis was carried out as per the following steps and was tabulated in the format illustrated in table 1.

Table 1: Tabulation method of $A B C$ analysis of drugs

\begin{tabular}{|c|c|c|c|c|}
\hline Name of the drug & Total cost (Rs) & Cumulative cost (Rs) & $\%$ cumulative cost & Category \\
\hline Drug A Drug B & $1,01,63,339.7367,70,883.50$ & $1,01,63,339.731,69,34,223.23$ & 2.89 & A \\
\hline & & & 4.82 & A \\
\hline
\end{tabular}

The annual consumption cost of the drugs in rupees was calculated by multiplying the annual demand with their unit price.

- The list was rearranged in the descending order of their annual consumption cost. An item with maximum value in rupees was at number one position and the item with least value appeared last in the list.

- The cumulative cost was worked out and shown against the item in the next column.

- The percentage of the cumulative cost was found to total consumption of all items and showed in the next column.

- Then the list was categorised into 3 groups-'A' 'B 'and 'C' based on annual consumption unit.

- "A" items: with highest money value $\mathbf{7 0 \%}$, represent only $10 \%$ of items
- "B" items: with medium money value $20 \%$, represent about $20 \%$ of items

- "C" items: withlowest money value $\mathbf{1 0 \%}$, represent about $70 \%$ of items

\section{Method II-graphical method}

A more accurate and practical method of doing $\mathrm{ABC}$ analysis is the graphical method.

- The percentage of the total cumulative value plotted on the vertical or Y-axis and the item number on the horizontal or X-axis.

- The number of items in the list influences the slope of the $A B C$ curve.

- On plotting the percentage of items used during the year on Xaxis and the value of annual consumption (as a percentage of the total) on the Y-axis a typical graph was obtained as shown in fig. 1.

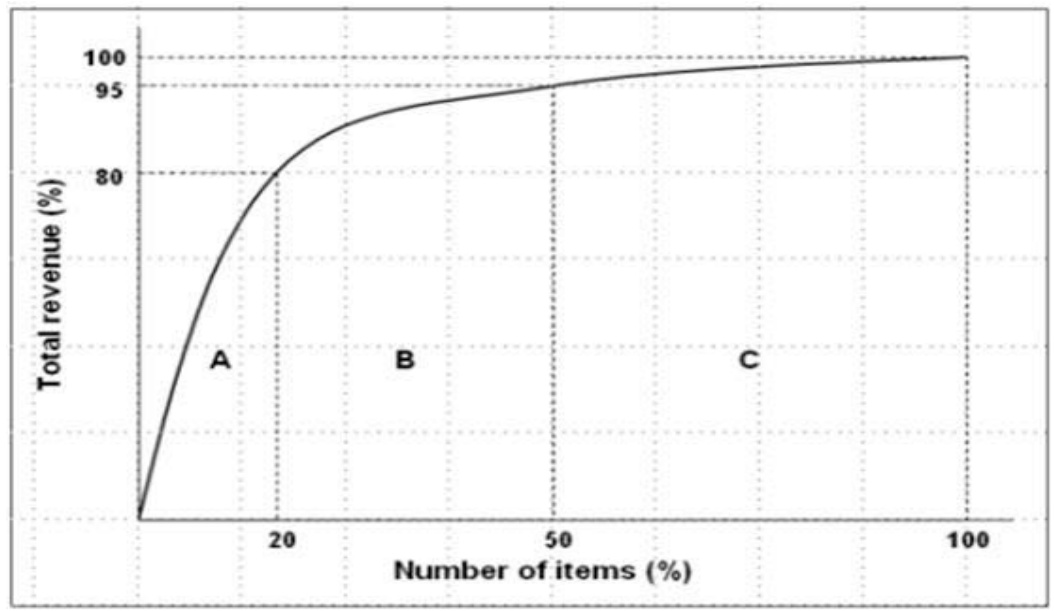

Fig. 1: Graphical method of ABC classification of drugs

Table 2: Calculation of HML analysis of drugs

\begin{tabular}{lll}
\hline Category of drugs & Description & Unit cost (Rs) \\
\hline $\mathrm{H}$ & High Priced Items & $\geq 500$ \\
$\mathrm{M}$ & Medium Priced Items & Between 500-100 \\
$\mathrm{L}$ & Low Priced Items & $<100$ \\
\hline
\end{tabular}




\section{High moderate low (HML) analysis}

HML analysis is similar to ABC analysis except for the difference that instead of Annual Inventory Turnover, cost per unit criterion is used. Items are tabulatedin descending order of unit cost as shown in table 2 [14-16].

\section{Assessment of knowledge of hospital pharmacists on methods of inventory control}

The knowledge of the hospital pharmacists on methods of inventory control was assessed using a self-developed questionnaire which was based on an original questionnaire developed by Harrison Lyombe T, et al. [17]. The original questionnaire consisted of 12 closed-ended and 7 open-ended questions. Open-ended questions were used to elicit experiences about inventory system that exist in the hospital. In the questionnaire, the respondents were made to give 'Yes" or "No" responses for questions on the application of ABC and VED. Apart from demographic data questions, a zero (0) point was given for an incorrect answer and one (1) point was given for the correct answer. The levels of knowledge were graded as poor $(0-$ $49 \%$ ), average (50-74\%) and good (75-100\%). Criteria used for ranking these levels were: participants who scored less or equal to 5 were ranked to be of poor knowledge, participants who answered correctly for 5 to 8 questions were designated as having average knowledge and lastly, participants who responded correctly for 9 to 12 questions were designated to have good knowledge.

\section{Modified questionnaire}

The modified questionnaire consisted of 12 questions in two parts. The first part (PART A) of the questionnaire consisted of 1 question with 5 subdivisions on the demographic details such as age, gender, educational qualification, job title and work experience of the participants. The second part (PART B) consisted of questions 2 to 12 as follows:

2. Are you aware of any inventory control methods practised in the central pharmacy of your hospital?

3. Your understanding of the purpose of the inventory control system in a hospital pharmacy is to understand

a. when to order stock; b. when to issue sock; c. how much stock to order; d. how much to issue; e. inventory level to maintain in order to avoid shortages

4. Are there written standard operating procedures for inventory management that you use?

5. Are you involved in assessing and estimating the quantities of medicines required per year?

6. What is the method adopted by your pharmacy in keeping records for inventory control management?

7. Where did you learn about inventory control techniques?
8. What is the method adopted by your pharmacy in keeping records for inventory control management?

9. Do you have any problems with using the above methods of keeping records?

10. How often do you experience out of stock situations during dispensing of drugs in a week?

11. Do you wish to implement any changes in the current method/change the inventory control technique in the pharmacy?

12. Are you willing to get additional training regarding inventory control techniques?

Each correct answer was given a score of 1 and an incorrect answer was given 0 . Participants with an average score of $\leq 5$ were ranked to be of poor knowledge; with a score of $>5$ and $\leq 8$ were categorised as having average knowledge and participants with a score of $>8$ and 11 were designated to have good knowledge. The levels of knowledge were graded as poor (0-49\%), average (50-74\%) and good (75$100 \%)$ based on the scoring. This self-developed questionnaire was validated by two subject experts and also statistically validated by using Cohen's kappa method. The Cohen's kappa value obtained was 0.7 which was in the range of 0.6 to 0.8 indicating that there is a substantial agreement between the two raters on the relevance of the questionnaire to the objective of the study.

\section{Statistical analysis}

Data on ABC and HML analyses were done using MS Excel 2010 Statistical functions and the results were expressed as descriptive statistics. IBM Statistical Package for Social Sciences (SPSS) version 23.0 software was used for statistical analysis. Chi-square test was done to assess the association between the cost of the drugs and the quantity purchased during the financial year 2017-2018. Pearsons' correlation analysis was done to find if there is any correlation between the characteristics like pharmacist's age/gender/educational qualification/work experience and their knowledge on the inventory control techniques. A $\mathrm{P}$ value of less than 0.05 was considered statistically significant.

\section{RESULTS}

During the studied financial year 2017-2018, the hospital's central pharmacy had purchased and stocked 4057 drugs in different doses, formulations and brands.

\section{Always better control (ABC) analysis}

Of 4057 drugs, about 330 drugs were classified under "A" category according to $8.13 \%$ of the total number of drugs purchased but incurred $70.91 \%$ of total annual consumption money value. The category "B" consisted of 667 drugs accounting to $16.44 \%$ of the total number of drugs and cost up to $20.01 \%$ of total annual money consumption. Category " $\mathrm{C}$ " had 3060 drugs which accounted to $75.42 \%$ of the total number of drugs purchased, which reflected only on $9.01 \%$ of annual money consumption as shown in table 3 and fig. 2 .

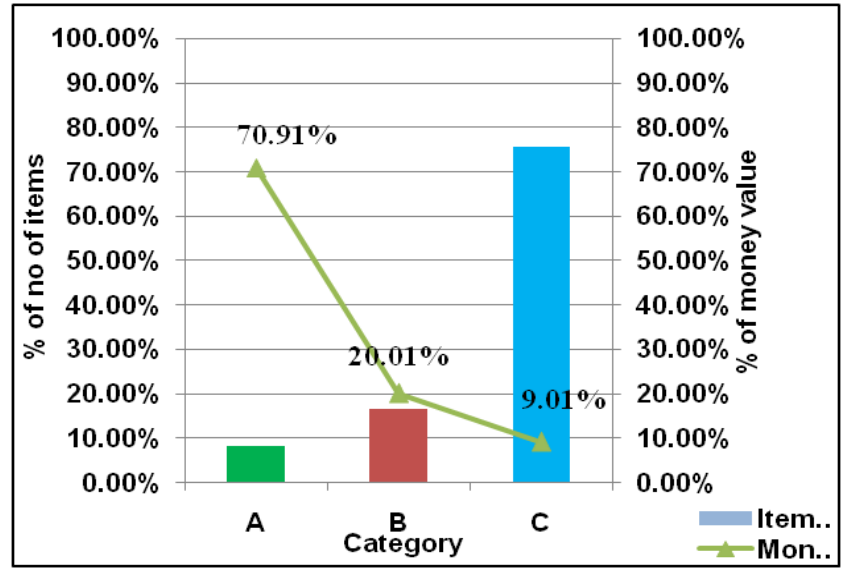

Fig. 2: Graphical representation of $\mathrm{ABC}$ analysis of drugs 
Table 3: ABC analysis of drugs

\begin{tabular}{|c|c|c|c|c|c|}
\hline \multirow[t]{2}{*}{ S. No. } & & \multicolumn{4}{|c|}{ Category } \\
\hline & & A & B & C & Total \\
\hline 1 & Total annual consumption (\%) & 70.91 & 20.01 & 9.01 & 100 \\
\hline 2 & No of items & 330 & 667 & 3060 & 4057 \\
\hline 3 & Percentage of items & 8.13 & 16.44 & 75.42 & 100 \\
\hline
\end{tabular}

\section{High, moderate low (HML) analysis}

Table 4 and fig. 3 indicate that of total 4057 drugs, 440 drugs (10\%) had a unit price above Rs. 500 and so were classified under ' $\mathrm{H}$ ' category. The drugs with unit price between Rs.500 and Rs.100 were grouped as ' $M$ ' items. There were 695 drugs $(17.13 \%)$ in ' $M$ ' category. The 'L' category consisted of 2922 drugs (72.02\%) with their unit price below Rs.100.

\section{Table 4: HML categorization of drugs}

\begin{tabular}{lll}
\hline Category & No. of items (N=4057) & Items \% \\
\hline High & 440 & 10.84 \\
Moderate & 695 & 17.13 \\
Low & 2922 & 72.02 \\
\hline
\end{tabular}

Based on chi-square analysis, there was no statistically significant association between the cost and the number of drugs purchased in the central pharmacy during the financial year of 2017-2018 $(\mathrm{P}=0.67)$.

\section{Assessment of knowledge of hospital pharmacists on methods of inventory control}

Table 5 depicts the demographic characteristics of the 80 study participants. Of the study participants, $20(25 \%)$ were males and $60(75 \%)$ were females. $26(32 \%)$ participants were in the age group of 20-29 y, $39(49 \%)$ were in the range of 30-39 y and $15(19 \%)$ were in the age group of 40-49 y. Education level was assessed in which 61(76\%) were with D. Pharmacy qualification, 15 participants (19\%) were with B. Pharmacy and 4 participants (5\%) was with Pharm. D./M. Pharmacy/MBA qualification. There were $73(91 \%)$ study participants with the job title as Pharmacist and $7(9 \%)$ as Pharmacist-intern. There were $10(13 \%)$ participants with work experience $<2$ y and $11(14 \%)$ with $2-5$ y, $18(22 \%)$ with $5-7 \mathrm{y}$ and $4-1(51 \%)$ with $>7 \mathrm{y}$ in the central pharmacy.

Table 5: Demographics of the study participants

\begin{tabular}{lll}
\hline Characteristics & No. of participants (N= 80) & \% \\
\cline { 2 - 3 } & $\mathbf{n}$ & 25 \\
\hline Gender & 20 & 75 \\
Males & 60 & 32 \\
Females & & 49 \\
Age in years & 26 & 19 \\
$20-29$ & 39 & 76 \\
$30-39$ & 15 & 19 \\
40-49 & & 5 \\
Educational level & 61 & 91 \\
D. Pharmacy & 15 & 09 \\
B. Pharmacy & 4 & 13 \\
Job title & & 14 \\
Pharmacist & 73 & 22 \\
Pharmacist-intern & 7 & 51 \\
Work experience (years) & & \\
2-5 & 10 & \\
5-7 & 11 & \\
$>7$ & 18 & \\
\hline
\end{tabular}

Table 6 indicates the response given by the participants for the questions 2 to $5,9,11$ and 12 . Majority of the pharmacists (98\%) were aware of the inventory methods, the purpose of the inventory. Although $74 \%$ of participants were aware of the availability of written standard procedure for inventory management, only 59\% were involved in assessing and estimating the quantities of medicines required per year. $74 \%$ of participants stated that they had no problems in record keeping and $61 \%$ wished to implement changes in the current method of inventory control. $74 \%$ of participants expressed interest to acquire additional training regarding inventory control techniques.

Table 6: Assessment of overall knowledge of participants

\begin{tabular}{|c|c|c|c|}
\hline Question No. & Question & Yes & No \\
\hline 2 & Are you aware of any inventory control methods practised in the central pharmacy? & 78 & 2 \\
\hline 3. a & The purpose is to understand when to order stock & 69 & 11 \\
\hline 3. b & The purpose is to understand when to issue stock & 69 & 11 \\
\hline 3. c & The purpose is to understand how much stock to order & 78 & 2 \\
\hline 3. $d$ & The purpose is to understand how much to issue & 72 & 8 \\
\hline 3. e & The purpose is to understand inventory level to maintain in order to avoid shortages & 79 & 1 \\
\hline 4 & Is there written the standard procedure for inventory management that you use? & 59 & 31 \\
\hline 5 & Are you involved in assessing and estimating the quantities of medicines required per year? & 47 & 33 \\
\hline 9 & Do you have any problems in keeping records? & 21 & 59 \\
\hline 11 & Do you wish to implement changes in the current method of inventory control? & 31 & 49 \\
\hline 12 & Do you wish to acquire additional training regarding inventory control techniques? & 59 & 21 \\
\hline
\end{tabular}


Of 80 study participants, 66 answered as HML, 14 answered as ABC for the question no.6 (What is the inventory control methods practised in the central pharmacy of your hospital?); 64 (80\%) answered as on job and 4 answered as academic training for the question no.7 (Where did you learn about the inventory control techniques?); 64 answered as Electronic drug record system, 10 answered as Ledgers and 6 answered as Bin cards for the question no.8 ("What is the method adopted by your pharmacy in keeping records for the inventory control management?); 54 answered as 15 times in a week and 26 answered as 5-10 times in a week for the question no.10 (how often do you experience out of stock situations during dispensing of drugs in a week?).

Table 7 shows that of 80 study participants, 64 (80\%) had an overall score of $>8-11$. The score was between 6 -8 for 12 participants $(15 \%)$ and $\leq 5$ for $4(5 \%)$ participants. Of the 59 participants $(74 \%)$ who were willing to acquire additional training on inventory control techniques, 46 participants had good overall knowledge, 10 participants had average overall knowledge and 3 participants had poor overall knowledge on the inventory control techniques.

Table 7: Overall knowledge score of study participants

\begin{tabular}{llll}
\hline Score range & $\begin{array}{l}\text { No of study participants } \\
(\mathbf{N}=\mathbf{8 0})\end{array}$ & Overall knowledge \\
\cline { 2 - 4 } & $\mathbf{N}$ & 5 & Poor \\
\hline$\leq 5$ & 4 & 15 & Average \\
$6-8$ & 12 & 80 & Good \\
$>8-11$ & 64 & 5 & \\
\hline
\end{tabular}

Table 8 indicates that there was a statically significant association between the education, work experience of the study participants and their knowledge on inventory control techniques whereas age and gender had no significant influence over their knowledge on inventory analysis.

Table 8: Baseline characteristics Vs Knowledge of inventory control techniques

\begin{tabular}{lll}
\hline Baseline characteristics & Chi square value & Significance 'P' \\
\hline Gender & 0.068 & 0.8 \\
Age & 1.002 & 0.61 \\
Education & 11.269 & $0.004^{* *}$ \\
Work experience & 8.710 & $0.03^{*}$ \\
\hline
\end{tabular}

*A P value of $<0.05$ was considered significant; ${ }^{* *} \mathrm{P}<0.01$ was considered highly significant

\section{DISCUSSION}

In health care system, the hospitals are dynamic institutions where utilizing the availability of drugs is the topmost priority and this cannot be achieved by focussing only on one factor whether cost or criticality [16]. When cost is the main focus of the inventory, less costly but vital drugs may be missed, whereas if the focus is on both cost and criticality, better monitoring of the inventory would be achieved and situations like drug less periods may be avoided.

Inventory at the hospital have to be maintained to sustain critical patient care activity and at the same time, a balance has to be maintained with an optimum inventory with minimal investment of the capital. There are various methods to maintain an inventory stock in the hospital such as Vital, Essential, Desirable (VED), ABC, ABC-VED matrix, HML etc. The major role of $\mathrm{ABC}$ and $\mathrm{HML}$ analysis is to assess the cost of the drugs and not onthecriticality of the drugs. But VED helps to assess the criticality of drugs in inventory control [18].

The study done by Sukhbir Singh, et al. [1], concluded that the ABC and VED techniques should be adopted for optimal and rational use of resources and elimination of out-of-stock situations in the hospital pharmacy.

In the present study, both $\mathrm{ABC}$ and HML analysis were used to study their applicability and efficiency as an inventory control method in the central pharmacy of a 2280 bedded, tertiary care teaching hospital.

As stated in the previous literature, the reason for choosing the $\mathrm{ABC}$ analysis in the present study is that the $A B C, A B C-V E D$ matrix method identifies the drugs requiring stringent control for optimal use of funds and avoid out of stock situations in the medical store. HML analysis is the inventory control technique which is easy to apply and calculate but to our knowledge, there is no literature on usage of HML analysis in the pharmacy store as widely published as the former methods.

In the present study, the central pharmacy had purchased 4057 drugs in the financial year 2017-2018. The study results showed that $330(8.13 \%)$ drugs consumed $70.91 \%$ of annual consumption and categorised as group " $A$ ". The category "B" consisted of $667(16.44 \%)$ drugs and costs up to $20.01 \%$. Category "C" had 3060 (75.42\%) drugs with a cost of $9.01 \%$ of the total value.

The obtained results were in accordance with the study conducted by Sukhbir Singh, et al. [1], on ABC, VED and ABC-VED matrix analysis of the pharmacy store of Post Graduate Institute of Medical Education and Research (PGIMER), Chandigarh, India, in which the study revealed that $11.08 \%, 22.16 \%$ and $66.75 \%$ items as $A, B, C$ category items respectively, accounting for $70.04 \%, 19.93 \%$ and $10.2 \%$ of annual drug expenditure of the pharmacy. The results obtained in the present study are also similar to studies conducted by Mitra S, et al. [11], and Anand T, et al. [13].

There is a need for strict managerial control, accurate data-driven forecasting of demand, close check on budgetary control, minimum safety stock, staggered purchase orders, frequent stock taking and judicious purchasing, stocking, issue and inspection policy for category A drugs. Category B drugs require moderate control over safety stock, purchasing and inspection. There is a minimal need for control measures of order and purchase of category $C$ drugs. ABC analysis alone helps selectively in controlling inventory on annual usage value alone, based on cost neglecting the criticality factor which is not acceptable in medical practice. Thus selective inventory control of medical stores integrates the dual concept of usage and criticality for better and more patient-centric control of drug inventory [18].

HML analysis is the classification of drugs based on its unit price. In this study of total 4057 drugs, 440 drugs (10\%) had a unit price above Rs.500 and so were classified under ' $\mathrm{H}$ ' category. The drugs with unit price between Rs.500 and Rs.100 were grouped as 'M' items. There were 695 drugs $(17.13 \%)$ in ' $M$ ' category. The ' $L$ ' category consisted of 2922 drugs (72.02\%) with their unit price below Rs.100.

The present study revealed that most of the drugs in the $\mathrm{H}$ category in HML analysis was also categorised as A in ABC analysis. Also found that there was no statistically significant association between the cost of the drugs and the number of drugs purchased in the central pharmacy. 
The inventory control methods are helpful to avoid overstocking and to calculate the safety stock [19]. Overstocking may lead to medicines pilling up at the health facility and eventually expiring while understocking of life serving medicines may lead to loss of lives. Keeping a safety stock is important as it helps to maintain the service level and for medicines, it is even more important since one would not want to have patients go without medications. Thus selective inventory control of medical stores integrates the dual concept of usage and criticality for better and more patient-centric control of drug inventory [20].

For the effective application of stringent inventory management controls, it is essential that the personnel working and involved in the inventory control have good knowledge about the methods and purpose of inventory control. Hence this study made an attempt to assess the knowledge of the hospital pharmacist working in the central pharmacy.

A self-developed questionnaire which was statistically validated was used to assess the overall knowledge on the methods of inventory control methods. 80 willing hospital pharmacists were included as the study population. The study showed that of 80 study participants $64(80 \%)$ had an overall score of above 8 with good overall knowledge of the inventory techniques. 12 participants $(15 \%)$ had a score of between 6-4 indicating that they had an average overall knowledge and 4 participants (5\%) had a score of5 indicating that they had poor overall knowledge.

The present study revealed that $80 \%$ of the study population had good overall knowledge on the inventory control methods and 59 participants were willing to acquire additional training on inventory control techniques though a majority of them had good knowledge. The present study also found that there was a statistically significant association between the participants' educational qualification, work experience and their knowledge on inventory control methods.

A similar study to assess the knowledge of the personnel involved in the inventory control was carried out by Godeliver A B Kagashe, et al.[21] and the study concluded that the poor knowledge of the personals was one of the factors which contributed to stock-outs in a public hospitals of Dar Es Salaam region in Tanzania and the author suggested that the personals should be provided training on inventory control techniques.

\section{LIMITATIONS}

In the present study, VED analysis was not included, because it was difficult to establish vital, essential and desirable drugs among 4057 drugs in the central pharmacy. But this method can be effective if utilized for stringent control of drug resources.

The ABC and HML analysis was done only for drugs. Inclusion of the surgical products for inventory control analysis would have given a clear picture of the inventory control techniques.

\section{CONCLUSION}

The study explained the need for the application of $\mathrm{ABC}$ and $\mathrm{HML}$ analysis, and the involvement of hospital pharmacists in the regular implementation of inventory analysis towards effective management of Pharmacy stores in a hospital setting. This would facilitate identifying the items belonging to important categories, and decision making in the purchase and distribution of specific items. Close supervision of the drugs requiring rigorous control leads to the rational use of resources and elimination of out-of-stock situations in the hospital pharmacy.

\section{ACKNOWLEDGMENT}

We thank all the volunteers participated in the study. Our sincere gratitude is extended to the Management of Sri Ramachandra Medical Center and Hospital for the constant support provided

\section{AUTHORS CONTRIBUTIONS}

All the authors have contributed equally in designing the study, obtaining ethical consent, data collection, recording, analysing and manuscript preparation.

\section{CONFLICT OF INTERESTS}

Authors declare no conflict of interest

\section{REFERENCES}

1. Singh S, Gupta AK, Latika, Devnani M. ABC and VED analysis of the pharmacy store of a tertiary care, academic institute of the Northern India to identify the categories of drugs needing strict management control. J Young Pharm 2015;7:76-80.

2. Kant S, Pandaw CS, Nath LM. A management technique for effective management of medical store in hospitals. Medical store management technique. J Acad Hosp Adm 1997;8-9:41-7.

3. Kunders GD, Gopinath S, Katakam A. Planning and designing supportive services-pharmacy. Hospitals: planning, design and management. New Delhi: Tata McGraw-Hill Publishing Company Limited; 2000. p. 273-81.

4. Gupta S, Kant S. Inventory control. Hospital stores management-an integral approach. New Delhi: Jaypee Brothers Medical Publishers; 2000. p. 60-72.

5. Thawani VR, Turankar AV, Sontakke SD, Pimpalkhute SV, Dakhale GN, Jaiswal KS, et al. Economic analysis of drug expenditure in Government Medical College Hospital, Nagpur. Indian J Pharmacol 2004;36:15-9.

6. Beier FJ. The management of the supply chain for hospital pharmacies: a focus on inventory management practices. J Business Logistic 1995;16:153-77.

7. Temeng VA, Eshuan PA, Essey PRK. Application of inventory management principles to explosive products manufacturing and supply-Acase study. Int Res J Finance Economics 2010;38:198-209.

8. West D. Purchasing and inventory management. In: Desselle SP, Zgarrick DP. editors. Pharmacy management: essentials for all practice settings. $2^{\text {nd }}$ ed. New York: McGraw-hill co, Inc.; 2009. p. 383.

9. Ceylan Z, Bulkan S. Drug inventory management of a pharmacy using ABC and VED analysis. Eur J Health Technol Assessment 2017;2:13-8.

10. Gandhi P, Basur A. Application of $A B C$ analysis in medical stores of ESIC, Delhi. Health Adm 2000;10:90-5.

11. Mitra S, Pattanayak S, Bhowmik P. Inventory control using ABC and HML analysis-a case study on a manufacturing industry. Int J Mechanical Industrial Eng 2013;3:76-81.

12. Nursing Management: ABC, VED, HML analysis. Currentnursing.com; 2011. Available from: http://currentnursing com/nursing_management/material_management_ABC_VED_HML _analysis.html. (Last accessed on 25 Mar 2017]

13. Anand T, Ingle G, Kishore J, Kumar R. ABC-VED analysis of a drug store in the department of community medicine of a medical college in Delhi. Indian J Pharm Sci 2013;75:113.

14. Gopalakrishnan P, Sundaresan M. Material management: an integrated approach. Practice hall, New Delhi; 1995. p. 60-72.

15. Devnani M, Gupta AK, Nigah R. ABC-VED analysis of the pharmacy store of a tertiary care teaching, research and referral health care institute of India. Pharm Management 2010;2:201-5.

16. Gupta R, Gupta KK, Jain BR, Garg RK. ABC and VED analysis in medical stores inventory control. Med J Armed Forces India 2007;63:325-7.

17. Harrison Lyombe T. Analysis of medicines expenditures and pharmaceutical inventory control management at Muhimbili National Hospital; 2013. p. 1-77.

18. Sushil Kumar M, Chakravarty B. ABC-VED analysis of expendable medical stores in a tertiary care hospital. Med J Armed Forces India 2014;71:24-7.

19. Satibi S, Putu Satrya Dewi DA, Akhmad AD, Kaswindiarti N, Puspandari DA. Drug cost analysis on national health insurance era in health support facilities. Asian J Pharm Clin Res 2017;10:119-21.

20. NurolainiKifli, Wong Wai See, Li Ling Chaw. The extent of medicine wastages in a tertiary hospital in Brunei: $10 \mathrm{y}^{\prime}$ retrospective study from 2006 to 2016. Asian J Pharm Clin Res 2018;11:328-32.

21. Kagashe G AB, Massawe T. Medicines stock out and inventory management problems in public hospitals in tanzania: a case of dares salaam region hospitals. Int J Pharm 2012;2:252-9. 